\title{
Different response to eccentric and concentric training in older men and women
}

\author{
Matthias Mueller · Fabio Andreas Breil · Michael Vogt • \\ Roger Steiner · Kurt Lippuner • Albrecht Popp • \\ Stephan Klossner · Hans Hoppeler · Christoph Däpp
}

Accepted: 3 June 2009 / Published online: 20 June 2009

(C) Springer-Verlag 2009

\begin{abstract}
Sarcopenia is the age-related loss of muscle mass and strength and has been associated with an increased risk of falling and the development of metabolic diseases. Various training protocols, nutritional and hormonal interventions have been proposed to prevent sarcopenia. This study explores the potential of continuous eccentric exercise to retard age-related loss of muscle mass and function. Elderly men and women $(80.6 \pm 3.5$ years $)$ were randomized to one of three training interventions demanding a training effort of two sessions weekly for 12 weeks: cognitive training (CT; $n=16$ ), conventional resistance training (RET; $n=23$ ) and eccentric ergometer training (EET; $n=23$ ). Subjects were tested for functional parameters and body composition. Biopsies were collected from M. vastus lateralis before and after the intervention for the assessment of fiber size and composition. Maximal isometric leg extension strength (MEL: $+8.4 \pm 1.7 \%$ ) and eccentric muscle coordination (COORD: $-43 \pm 4 \%$ ) were significantly improved with EET but not with RET (MEL: $+2.3 \pm 2.0 \%$; COORD: $-13 \pm 3 \%$ ) and CT (MEL: $-2.3 \pm$ $2.5 \%$; COORD: $-12 \pm 5 \%$ ), respectively. We observed a loss of body fat $(-5.0 \pm 1.1 \%)$ and thigh fat $(-6.9 \pm$ $1.5 \%)$ in EET subjects only. Relative thigh lean mass increased with EET $(+2.5 \pm 0.6 \%)$ and RET $(+2.0 \pm 0.3 \%)$
\end{abstract}

M. Mueller $(\bowtie) \cdot$ F. A. Breil $\cdot$ M. Vogt $\cdot$ R. Steiner $\cdot$ S. Klossner H. Hoppeler · C. Däpp

Institute of Anatomy, University of Bern, Baltzerstrasse 2, 3000 Bern 9, Switzerland

e-mail: matthias.mueller@ana.unibe.ch

K. Lippuner · A. Popp

Osteoporosis Policlinic, Inselspital, Bern University Hospital

and University of Bern, 3010 Bern, Switzerland and correlated negatively with type IIX/type II muscle fiber ratios. It was concluded that both RET and EET are beneficial for the elderly with regard to muscle functional and structural improvements but differ in their spectrum of effects. A training frequency of only two sessions per week seems to be the lower limit for a training stimulus to reveal measurable benefits.

Keywords Eccentric $\cdot$ Elderly $\cdot$ Strength $\cdot$ Fat . Coordination $\cdot$ Fibertyping

\section{Introduction}

Sarcopenia is a condition of muscle tissue characterized by the loss of muscle fibers and fiber atrophy (Doherty 2003; Lexell 1995) accompanied by increased infiltration of noncontractile components such as connective tissue and fat (Overend et al. 1993). These structural changes along with impaired neuronal functions result in loss of muscle strength (Skelton et al. 1994). The mechanisms underlying muscle atrophy and loss of innervation are not fully understood. Reduced physical activity, decline in anabolic hormone levels (dehydroepiandrosterone, testosterone, growth hormone) concomitant with a chronic low-grade inflammation (increased tumor necrosis factor $\alpha$ and cortisol serum levels) contribute to the loss of muscle mass (Doherty 2003; Vandervoort 2002). Improvement of leg strength by means of strength training is a broadly applied strategy to reduce the risk of falling, since these two parameters seem causally associated (Perry et al. 2007; Shigematsu et al. 2006). Heavy resistance training can successfully be applied in the elderly (Hruda et al. 2003; Wieser and Haber 2007). However, it can result in significant cardiovascular as well as substantial mechanical stress on single joints 
(Hungerford and Barry 1979; Kaufman et al. 1991). On the other hand, endurance ergometer training, carried out in a closed muscle chain at high-angular velocities, has a broad application, improving body composition and insulin sensitivity (Hersey et al. 1994), but with minor benefits for strength and muscle mass, as mechanical stress on muscles remains low in endurance cycling.

It is well documented that strength training in the elderly results in substantial strength gain and muscle hypertrophy (Kryger and Andersen 2007). A recent meta-analysis by Roig et al. (2008) evaluated 20 studies comparing eccentric and concentric strength training and concluded that subjects profited more from eccentric than concentric training with regard to strength gain and muscle hypertrophy. These studies compared maximal eccentric training protocols applied to young adults and trained athletes and draw attention to the prospects of eccentric exercise modalities. In view of potential benefits of eccentric exercise we explored a training protocol of continuous eccentric exercise and compared it to established conventional strength training protocols. The eccentric exercise training (EET) consisted of resisting the pedal movement of a motor driven recumbent ergometer. As the energy cost of eccentric work is approximately four times less than that of concentric work of a comparable external load (Lastayo et al. 1999) large torques can be exerted at a manageable metabolic cost. The ensuing low stress on the cardiovascular system is particularly important for elderly, since they are characterized by a reduced aerobic capacity (Lotscher et al. 2007). The objective of using EET thus was to achieve a high mechanical load on muscle tissue with a restricted aerobic demand. Similar to concentric ergometer training, EET is executed in a closed muscle chain at relatively high-angular velocities, minimizing peak forces on single joints (Ericson and Nisell 1986, 1987). Due to these favourable features, eccentric exercise has been applied to people with a limited tolerance for conventional strength training (LaStayo et al. 2003), chronic obstructive pulmonary disease (Rooyackers et al. 2003) and coronary disease (Steiner et al. 2004). As a drawback, eccentric exercise is potentially associated with delayed onset muscle soreness (DOMS) due to muscle tissue damage (Friden et al. 1983). In the present study, muscle damage was avoided by carefully increasing muscle load over repeated exercise sessions beginning with very low eccentric loads.

It has been suggested that strength gain in the elderly is more related to neural mechanisms and less to muscle hypertrophy (Moritani and deVries 1980). Using an intensive exercise protocol, Kryger and Andersen (2007) still reported a significant increase of the type IIA fiber area and an impressive $37 \%$ strength gain in subjects, aged 85 and older, after a 12 week training period with three heavy resistance training sessions ( 45 min each) per week, indicating the maintenance of muscular plasticity on a structural as well as on a functional level even in very old people.

The aim of this study was to investigate EET as an alternative to conventional resistance training for the elderly to increase leg strength and leg muscle mass. This was done in a setting in which subjects were asked to perform only two sessions/week to maximize adherence. Based on the study of LaStayo et al. (Lastayo et al. 2002), we hypothesized that EET would be more effective in increasing muscle strength and mass than conventional resistance training. We further expected larger improvements of muscle strength in females than in males due to their initial lower fitness level (Lotscher et al. 2007). We did not expect any changes in muscle fiber type composition as the imposed training regime $(2 \times 20$ min effective training time per week for 12 weeks $)$ seemed to be insufficient to produce fiber type changes.

\section{Materials and methods}

Subjects and study design

A total of 62 subjects (71-89, average 80.6 years) with stable medication and health conditions were included in the study. Subjects with severe neuromuscular disease, instable coronary disease or severe hip or knee arthritis were excluded (Lotscher et al. 2007). The study was part of the National Foundation Program 53 "Musculoskeletal health and chronic pain" and carried out in accordance with the guidelines and the approval \#190/04 of the "Kantonale Ethische Kommission".

The training period lasted for 12 weeks and comprised two guided training sessions (45 min each) per week. In order to familiarize the subjects with the functional tests they were sham tested 2 weeks before the real testing procedure. Subjects from whom biopsies were collected were randomized to one of the physical intervention groups and all others were randomly distributed as follows:

1. Cognitive training (CT) consisted of computer-guided cognitive training. The subjects ( 10 women, 6 men) did not perform any physical training and served as a control to account for the influence of social aspects of the training sessions.

2. Conventional resistance training (RET) was performed by 23 subjects ( 13 women, 10 men). RET was carried out in a gym and comprised four exercises for the lower extremity (leg press, knee extension, leg curl, hip extension). The sessions consisted of a 10-min warmup with cardiovascular activation and gymnastics, 20 min training and 10 min cool-down with stretching. For the first six sessions the individual loads were set very low to familiarize subjects with the exercises. 
Exercises included three sets with ten repetitions and loads were gradually increased during this time. The subsequent sessions consisted of one warm up set and two sets with eight to ten repetitions. If subjects were able to do ten repetitions or more, the load was increased in the next session. The load was not increased if people suffered from DOMS as indicated by scores $\geq 3$ on a visual analog scale (VAS) (Langley and Sheppeard 1985) or when rating of the perceived exertion (RPE) of the whole training session was $>13$ according to BORG (Borg et al. 1987). VAS ranges from 0 to 10 , where 0 is no soreness and 10 is the highest perceived muscle soreness. DOMS ratings of subjects were in the interval between 0 and 4 .

3. Eccentric ergometer training (EET) was carried out by 23 subjects (13 women, 10 men) on a custom-built motor-driven ergometer (Meyer et al. 2003). The trainings started with a 10-min warm-up on a conventional ergometer with minimal loads (females $10 \mathrm{~W}$, males $20 \mathrm{~W}$ ) and closed with 10 min cool-down with stretching, while the actual EET lasted $20 \mathrm{~min}$. The initial load on the eccentric bike was set very low (females $30 \mathrm{~W}$, males $50 \mathrm{~W}$ ). Initially, subjects exercised for only $5 \mathrm{~min}$ to prevent severe DOMS. During the first sessions the training duration was gradually increased in 5-min steps until it reached $20 \mathrm{~min}$, before the imposed load was ramped. Load was ramped in consecutive sessions by $20 \%$ of the individual maximal power output achieved in the initial ergometer ramp test to exhaustion (Lotscher et al. 2007). Contraindications to increase the workload were the same as those for RET (DOMS; RPE).

Some of the subjects had to be partly or entirely excluded from physical tests due to illness such as herpes zooster (1 woman EET), appendicitis (1 woman CT), osteoporosis (1 woman CT), progressive morbus Alzheimer (1 woman CT, 1 woman RET) or injuries and persisting joint pain (1 man CT, 1 woman RET, 1 man RET, 1 man EET). Other subjects were excluded because they were unable to complete the required test (MEL: 1 women CT, 1 man RET, 1 woman EET) or because they were unable to follow the training protocol (1 woman EET was not able to dose the eccentric ergometer). Compliance in the sessions was secured by coaches (at least one coach per two subjects). Subjects attended on average $89 \pm 2 \%$ of the training sessions.

Subjects were specifically instructed to continue their usual diet. However, no written reports on the dietary regime were obtained.

Specific training loads

Changes of training loads were assessed by the comparison of the loads after the 3 weeks of habituation to those at the very end of the intervention for RET and EET. For RET the average loads of the four exercises were compared.

Timed up \& go and Berg balance scale

The Berg balance scale (BBS) (Berg et al. 1992) and the timed up \& go (TUG) (Shumway-Cook et al. 2000) are functional tests designed for elderly people in order to assess their risk of falling (Lotscher et al. 2007). These tests were carried out at the beginning and at the end of the training period.

Body composition, muscle biopsies

Whole body composition (lean and fat tissue mass) was determined by dual energy X-ray absorptiometry (DEXA) (QDR-4500A, Hologic Inc., Bedford, USA). Thigh was defined as the part from tuber ischiadicum to the distal end of the femur, whereas the leg included the distal section of the limb below the tuber ischiadicum. Fat and lean values of thighs and legs include right and left extremities.

Biopsies were taken from 27 subjects (RET: 6 women, 7 men; EET: 7 women, 7 men) using the Bergström technique (Bergstrom 1975) from the mid thigh position of the M. vastus lateralis before and after the 12-week training period in a resting state, $48-72 \mathrm{~h}$ after the last exercise bout. Pre- and post-biopsies were collected from the same leg with the incision approximately $2 \mathrm{~cm}$ apart. For ethical reasons muscle biopsies were taken from subjects of the physical intervention groups only. Muscle samples were immediately frozen in liquid nitrogen cooled isopentane and stored in liquid nitrogen until required for further analysis.

\section{Histochemistry}

Selective myofibrillar ATPase inactivation and subsequent staining was processed at $12 \mu \mathrm{m}$ cryostat cross-sections with preincubation at $\mathrm{pH} 4.5$ and 10.5 as described by Billeter et al. (1980). Fibers were classified as type I, type IIA and type IIX. The type II fiber population consists of the sum of the type IIA type IIX fibers. On average, 351 fibers were counted per biopsy. Reliability of the technique and the technician was assessed by the test-retest method with 10 randomly chosen biopsies. Pearson product-moment correlation coefficient was 0.95 . For the estimation of fiber type specific cross sectional areas a $30 \times 30 \mu \mathrm{m}$ grid was overlaid and points on fibers were counted. This procedure was applied in areas that appeared reasonably cross-sectioned ( 80 fibers per biopsy on average).

Maximal isometric extension of the legs (MEL)

Strength testing was performed as described by Lötscher et al. (2007). Subjects were fixed in a sitting position 
$\left(90^{\circ}\right.$ angle; ankle-knee-hip) on a force platform (Quattro Jump, Kistler Instrumente AG, Winterthur, Switzerland). They were verbally encouraged to push maximally against the platform for about $4 \mathrm{~s}$. The force was permanently recorded with a resolution of $500 \mathrm{~Hz}$. The best trial out of three was evaluated by determining the highest mean force over a one-second period. Normalization to the subject's body mass resulted in relative MEL.

\section{Eccentric coordination}

Estimation of eccentric coordination was carried out on the eccentric ergometer (Meyer et al. 2003). This parameter estimates a subject's ability to adjust the power of braking the pedals to the eccentric target load [W]. The appropriate load is self-monitored on a screen where the actual and the target load are graphically displayed in "real time" (Fig. 1). The deviation of actual from target load is estimated by the Root Mean Square (RMS; indicated by the hatched area in Fig. 1). The target load in the testing was set to $30 \mathrm{~W}$ for females and $50 \mathrm{~W}$ for males; subjects were tested over a 5-min period. In the EET group the post-test for eccentric coordination was carried out at the individual training load of each subjects' last training session (Table 1,2).

Data analysis

Data are presented as mean \pm SE. Interaction of training modality (CT, RET, EET) on functional parameters such as TUG, BBS, MEL and eccentric coordination was verified with an analysis of variance (ANOVA) for repeated measures and Tukey's Honest Significant Difference (HSD) post hoc test. For the ANOVA, the level of significance was set to $P<0.05$ and marked with $*$ and $* *$ for $P<0.01$, respectively. $P$-values in the figures are from Tukey's HSD and indicate the probability that differences between pre and post-intervention occur randomly. Analysis of sex specific improvements in MEL was analyzed in the eccentric group only using ANOVA with repeated measures. For the comparison of pre-post differences between groups we applied a Kruskal-Wallis ANOVA and verified significant results $(P<0.05)$ with Mann-Whitney $U$ post hoc testing
Fig. 1 Schematic set-up of the eccentric ergometer and evaluation of eccentric coordination. Root mean square $(R M S)$ represents the hatched area comprised by the target load and the executed power. The smaller the RMS, the better the eccentric coordination. Representative illustrations of pre- and post-test graphs from EET subjects. Note the difference of the match of the executed power and the target load in the post-test

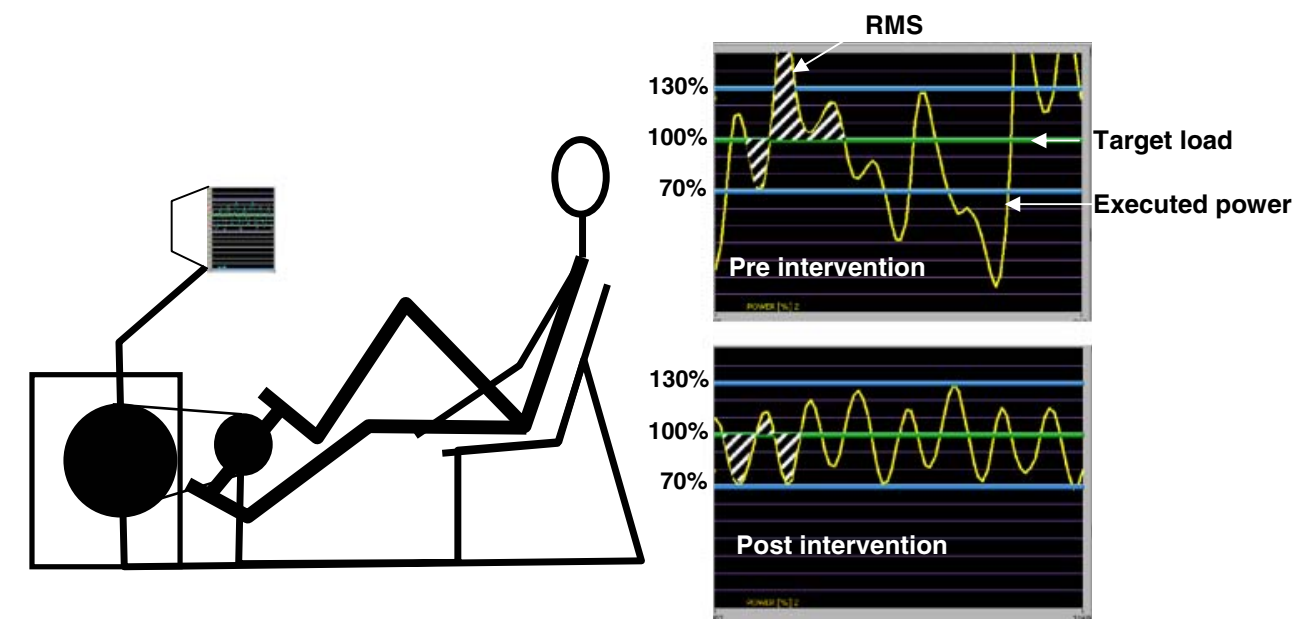

Table 1 Anthropometric characteristics of the study subjects

\begin{tabular}{|c|c|c|c|c|c|}
\hline & Women (36) & Men (26) & CT (16) & RET (23) & EET (23) \\
\hline Age (years) & $80.4 \pm 0.6$ & $80.9 \pm 0.6$ & $81.8 \pm 0.8$ & $80.1 \pm 0.8$ & $80.3 \pm 0.7$ \\
\hline Height $(\mathrm{cm})$ & $161 \pm 1$ & $176 \pm 1$ & $166 \pm 3$ & $167 \pm 2$ & $168 \pm 2$ \\
\hline$V \mathrm{O}_{2 \max }(\mathrm{ml} / \mathrm{min})$ & $1210 \pm 40$ & $1940 \pm 90$ & $1380 \pm 150$ & $1640 \pm 110$ & $1500 \pm 80$ \\
\hline Body mass (kg) & $65.5 \pm 1.9$ & $71.1 \pm 1.7$ & $70.5 \pm 2.8$ & $67.7 \pm 2.6$ & $66.1 \pm 1.8$ \\
\hline BMI $\left(\mathrm{kg} / \mathrm{m}^{2}\right)$ & $25.3 \pm 0.7$ & $23.1 \pm 0.6$ & $25.6 \pm 0.9$ & $24.3 \pm 1.0$ & $23.5 \pm 0.6$ \\
\hline Lean $(\mathrm{kg})$ & $42.7 \pm 1$ & $53.6 \pm 1.3$ & $48.4 \pm 2.5$ & $47.3 \pm 1.7$ & $46.5 \pm 1.6$ \\
\hline Fat (kg) & $21.0 \pm 1.3$ & $15.4 \pm 1.1$ & $18.9 \pm 1.3$ & $19.1 \pm 2.0$ & $17.6 \pm 1.3$ \\
\hline Fat rel $(\%)$ & $31.7 \pm 1.1$ & $21.2 \pm 1.3$ & $29.3 \pm 2.1$ & $26.7 \pm 1.9$ & $26.5 \pm 1.6$ \\
\hline
\end{tabular}

Parameters are displayed as mean values $\pm \mathrm{SE} . V \mathrm{O}_{2 \max }=$ maximal oxygen uptake, $\mathrm{BMI}=$ body mass index, Lean $=$ whole body lean content, Fat $=$ whole body fat content, Fat rel = relative whole body fat content. Mean values did not differ among groups (ANOVA) but among sex (all except age) according to a two tailed student's $t$ test $(P<0.05)$ 
Table 2 Estimation of subjects' fiber type composition: parameters are displayed as mean values $\pm \mathrm{SE}$

\begin{tabular}{|c|c|c|c|c|}
\hline & \multicolumn{2}{|l|}{ EET } & \multicolumn{2}{|l|}{ RET } \\
\hline & Pre & Post & Pre & Post \\
\hline \multicolumn{5}{|c|}{ Number $(\%)$} \\
\hline Type I & $55.2 \pm 3.3$ & $57.5 \pm 2.2$ & $52.3 \pm 3.7$ & $54.3 \pm 3.9$ \\
\hline Type IIA & $34.4 \pm 2.0$ & $35.6 \pm 1.9$ & $32.7 \pm 1.6$ & $31.4 \pm 2.4$ \\
\hline Type IIX & $10.3 \pm 2.0$ & $6.9 \pm 1.6$ & $15.1 \pm 4.2$ & $14.2 \pm 4.9$ \\
\hline \multicolumn{5}{|l|}{ Area $(\%)$} \\
\hline Type I & $62.9 \pm 2.7$ & $63.1 \pm 2.5$ & $56.6 \pm 3.3$ & $56.5 \pm 3.4$ \\
\hline Type IIA & $31.6 \pm 2.2$ & $32.6 \pm 2.3$ & $34 \pm 1.7$ & $35.2 \pm 2.0$ \\
\hline Type IIX & $5.5 \pm 1.2$ & $4.2 \pm 1.1$ & $9.3 \pm 3.3$ & $8.4 \pm 3.7$ \\
\hline \multicolumn{5}{|c|}{ Area $\left(\mu \mathrm{m}^{2}\right)$} \\
\hline Type I & $4,250 \pm 210$ & $4,392 \pm 200$ & $4,050 \pm 240$ & $4,030 \pm 270$ \\
\hline Type IIA & $3,890 \pm 340$ & $3,570 \pm 290$ & $3,990 \pm 490$ & $4,430 \pm 490$ \\
\hline Type IIX & $2,320 \pm 230$ & $2,220 \pm 210$ & $2,070 \pm 200$ & $1,950 \pm 160$ \\
\hline
\end{tabular}

Top panel displays individual fiber numbers in percentage, middle panel displays the cross sectional areas of the same fiber population in percentage of all fibers and the last panel shows the estimated average cross sectional area in square micrometers

(M-W $U$ test). The sex difference in eccentric coordination was verified with a two tailed student's $t$ test. The level of significance was set to $P<0.05$. Coefficients of correlation were calculated using Pearson Product Moment Correlation for pooled pre- and post-training datasets. All statistical analyses were carried out with the Statistica software package 6.1 (StatSoft (Europe) GmbH, Hamburg, Germany).

\section{Results}

Training specific improvements

Statistically significant improvements of the training loads could be recorded for RET and for EET subjects. RET subjects improved leg extension on average by $84.8 \%$ from $30.2 \pm 2.2$ to $55.8 \pm 3.7 \mathrm{~kg}$, while EET subjects increased average training load from $69.6 \pm 4.3$ to $314.8 \pm 27.0 \mathrm{~W}$ $(+352 \%)$. The increased training loads in EET overestimate the training progress since the subjects were ramped carefully to avoid muscle injury.

Timed up \& go (TUG), Berg balance scale (BBS)

Subjects improved significantly in TUG from $7.37 \pm 0.16$ to $6.88 \pm 0.16 \mathrm{~s}(-6.7 \pm 0.2 \%)$ independent of training modality (EET $-7.5 \pm 0.2 \%$; RET $-7.3 \pm 0.2 \%$; CT $-4.9 \pm 0.5 \%)$. No significant improvements could be recorded for BBS (EET $+1.7 \pm 0.3 \%$; RET $+0.7 \pm 0.3 \%$; $\mathrm{CT}+0.7 \pm 0.4 \%$ ) since the study-subjects had on average already achieved 53.8 of maximal 56 points in the pre-test.
Body composition

The EET group experienced a reduction in whole body fat $(-5.0 \pm 1.1 \%)$ and thigh fat content $(-6.9 \pm 1.5 \%)$ not observed in RET (body: $-0.6 \pm 1.0 \%$, thigh: $-2.7 \pm 0.9 \%$ ) and CT (body: $+1.4 \pm 1.2 \%$, thigh: $+0.6 \pm 1.9 \%$ ). Subjects' relative thigh muscle mass increased significantly with EET $(+2.5 \pm 0.6 \%)$ and RET $(+2.0 \pm 0.3 \%)$ but not with CT $(+0.4 \pm 0.4 \%)$ (Fig. 2). Pre-Post differences between RET and EET were verified for body $(P=0.002)$ and thigh fat $(P=0.03)$ by a Mann-Whitney $U$ test (M-W $U$ test).

Muscle fiber types

A significant reduction of the type IIX/type II ratio was recorded for EET subjects $(-22 \pm 14 \%)$ but not for RET subjects $(-8 \pm 14 \%)$ (Fig. 3a). Type IIX/type II fiber ratio correlated significantly $(P<0.01)$ with body composition parameters: positively with body fat $(R=0.75)$ and thigh fat $(R=0.70)$ and negatively with relative thigh lean mass $(R=0.69)$. Biopsies from women displayed a significantly higher type IIX/type II fiber ratio than biopsy samples from men (women: $29 \pm 5 \%$; men: $19 \pm 3 \%$; two-tailed students $t$ test, $P<0.05)$. Pre-post differences between RET and EET were not significant $(P=0.2 ; \mathrm{M}-\mathrm{W} U$ test $)$.

Maximal isometric extension strength of the legs (MEL)

The EET group significantly improved MEL $(+7.5 \pm 1.7 \%)$, whereas no significant strength changes were noticed for RET $(+2.3 \pm 2.0 \%)$ and CT $(-2.3 \pm 2.5 \%)$. Improvements of EET subjects were even more pronounced when MEL was normalized to body mass $(+8.4 \pm 1.7 \%)$ (Fig. 4). However, pre-post differences in MEL were not significant between EET and RET ( $P=0.1$; M-W $U$ test). Focusing on EET subject's relative MEL, exclusively women improved significantly (from $13.7 \pm 2.8$ to $14.4 \pm 2.8 \mathrm{~N} / \mathrm{kg} ;+13.8 \pm 2.5 \%$ ), whereas in men the observed increase was not significant (i.e. from $16.9 \pm 3.5$ to $17.2 \pm 3.6 \mathrm{~N} / \mathrm{kg} ;+4.8 \pm 1.4 \%$ ). The sex specific analysis was verified with a two tailed student's $t$ test with a level of significance offset at $5 \%$.

\section{Eccentric coordination}

The ability to match instantaneous muscle torque to eccentric target load was improved significantly by EET subjects $(-43 \pm 6 \%$ RMS $)$ but not by RET $(-13 \pm 3 \%)$ and CT subjects $(-12 \pm 5 \%)$ (Fig. 5). The initial inferior coordination performance of women compared to men (Pre: women: $79.6 \pm 7.3$ RMS; men: 55.4 \pm 6.7 RMS) was lost following EET (Post: women: $24.9 \pm 1.0$ RMS; men: $22.9 \pm 2.3$ RMS). Pre-Post differences between RET and EET were significant $(P=0.02)$ according to $\mathrm{M}-\mathrm{W} U$ test. 


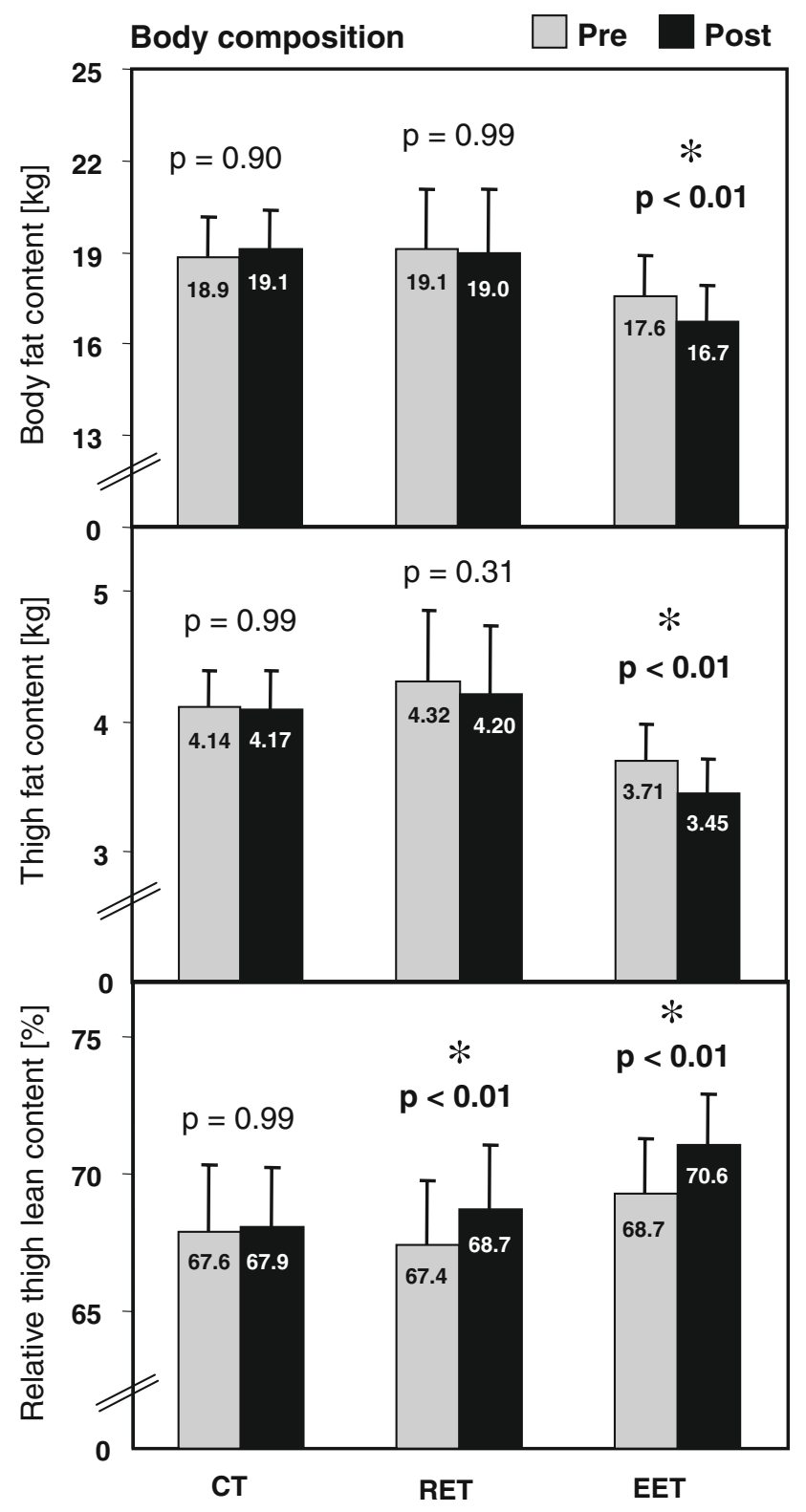

Fig. 2 Whole body fat, thigh fat and relative thigh lean content as assessed by dual energy X-ray absorptiometry. Bars (grey pre; black post) represent mean contents \pm SE in $\mathrm{kg}$ (fat) and \% (lean) of CT $(n=14)$, RET $(n=21)$ and EET subjects $(n=19)$. (ANOVA with repeated measures; $* P<0.05$; indicated $P$-values of Tukey's HSD Post hoc test)

\section{Discussion}

This study shows eccentric ergometer exercise and resistance training to be well tolerated by elderly. Despite a low training frequency of just two sessions per week we observed a moderately positive outcome. EET improved leg muscle strength, body composition and eccentric muscle coordination in elderly. The positive effects of RET
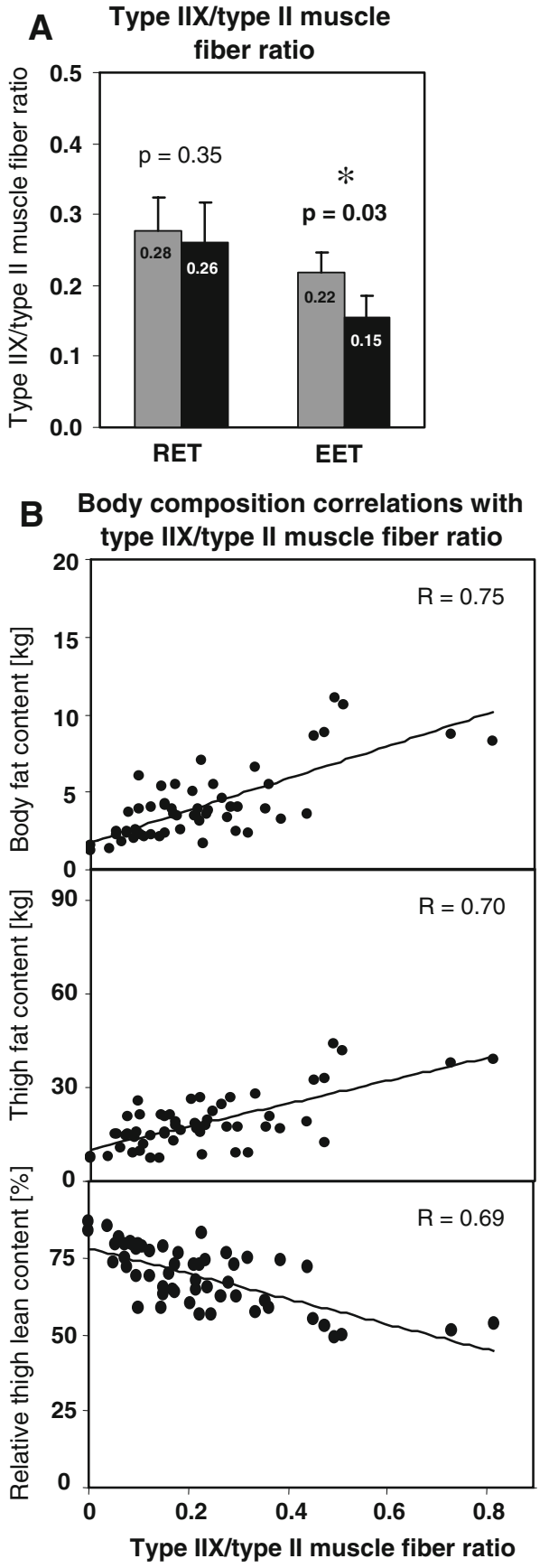

Fig. 3 a Ratio of type IIX/type II muscle fiber content of EET ( $n=14)$ and RET $(n=13)$. Bars represent means of pre- (grey) and post-values (black) \pm SE. (Indicated $P$-values of one tailed paired student's $t$ test; $* P<0.05)$. b Highly significant $(P<0.01)$ correlation of fibertype composition with body fat, thigh fat and relative thigh lean content. Data points consist of individual pre- or post-values of body composition parameters with the corresponding type IIX/type II muscle fiber ratio evaluated from muscle biopsies $(n=56)$

were similar in magnitude but mostly statistically not significant. The fact that strength gain in our study was measured on a training independent device underlines the task specific improvements in most other studies which don't necessarily reflect the "useful" benefits. 


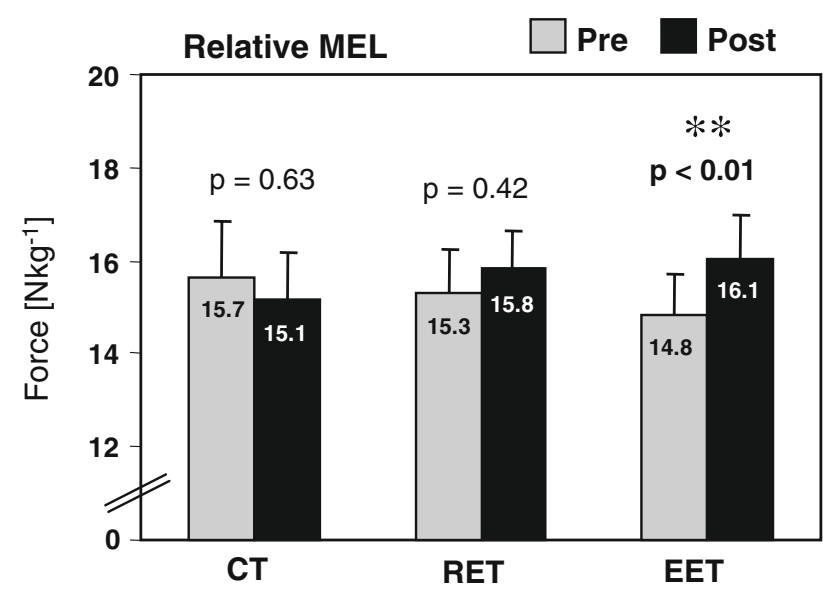

Fig. 4 Maximal isometric extension strength of the legs (MEL) normalized to body weight. Bars (grey pre; black post) represent mean leg force in $\mathrm{N} \mathrm{kg}^{-1}$ of CT $(n=13)$, RET $(n=18)$ and EET subjects $(n=19) \pm$ SE. (ANOVA with repeated measures; $* * P<0.01$; indicated $P$-values of Tukey's HSD Post hoc test)

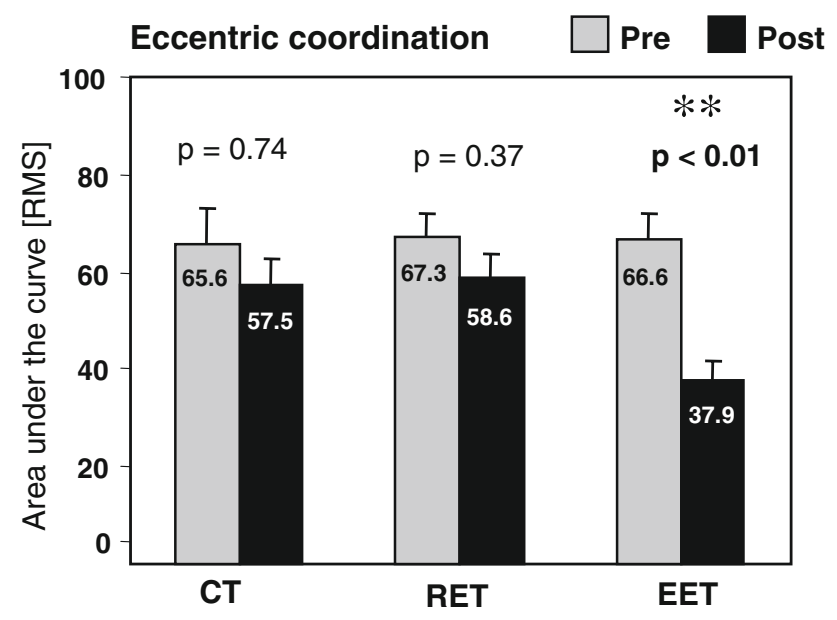

Fig. 5 Eccentric muscle work load as assessed on the eccentric ergometer. Bars represent root mean square $(R M S)$ of CT $(n=14)$, RET $(n=19)$ and EET $(n=19) \pm$ SE. (ANOVA with repeated measures; $* * P<0.01$; indicated $P$-values of Tukey's HSD Post hoc test)

A major limitation of our study was the low training frequency of our subjects which was chosen to maximize adherence for independently living volunteers, characterized by an active lifestyle and concomitant duties and obligations. Together with the exceptionally good physical condition of our subjects, functional tests such as TUG and BBS were not sensitive enough to detect differences. The modest increase in strength (as measured by MEL) seen in our study when compared to strength increases reported in other studies with elderly subjects (Hauer et al. 2001; Kryger and Andersen 2007) has several reasons: (1) our subjects were already in exceptional physical condition. (2) We used a low training frequency with just two sessions of 20 min specific training per week. We believe this to be a realistic sustainable training program for this population. (3) Our force measurement setting was devised to mimic an every day situation and thus independent from the training procedure for both training modalities. The randomisation procedure had to be adapted because not all of our subjects agreed to get biopsied. Due to medical reasons (i.e. use of anticoagulants) we could not biopsy some of the subjects. Subjects who fulfilled biopsy criteria were then randomized to one of the physical intervention groups (RET, EET). We did not collect biopsies from CT subjects as this would have represented an unwarranted risk. Since there were no significant initial functional differences between the groups we think it unlikely that the de facto stratified randomisation influences our results.

A major concern in this study was to avoid the negative consequences of eccentric exercise, consisting of muscle damage i.e. DOMS (Friden et al. 1983). To this end, we chose very low initial training loads $(30$ and $50 \mathrm{~W}$ for women and men, respectively) and short training times (5 min). Reported discomfort after eccentric exercise was thus, between 0 and 1 and never exceeded a value of 4 on a VAS scale of $0-10$. The prerequisite for safe eccentric exercise is to tailor and monitor eccentric load individually. This was achieved with a computer-based visual feedback system in which subjects were matched the instantaneous training load to a target load displayed on a computer screen (Fig. 1). Matching of the training load to a target load turned out to be a demanding coordination task which required coaching and some practice and turned out to be feasible for all except one subject. The software for our eccentric ergometer allows for quantitative assessing the deviation of the eccentric performance from the required load (eccentric coordination; see Fig. 1). Not surprisingly, only EET-trained subjects were able to improve eccentric coordination (by 43\%) significantly over the entire training period. The initial difference in coordination between better performing males than females was lost after training, as women improved their eccentric coordination more than men. It is currently difficult to assess the relevance of the massive improvement of eccentric coordination as defined in our setting. Matching eccentric performance to a target load is a complex task involving the integration of visual feedback with motor control. In a previous study using the same eccentric ergometer on world cup level alpine skiers, a positive correlation was found between the eccentric coordination and success in ski slalom races (Vogt et al. 2003). Whether improvements in eccentric coordination results in a lowered risk of falling needs further evaluation.

In the current study, the risk of falling was assessed by standard tests such as TUG and the BBS. All our subjects ranked in the lowest risk category at the outset of the study. For TUG, subjects performing the task in less than $14 \mathrm{~s}$ belong to the category of people with no increased risk of 
falling. The subjects in our study performed this task in the pre-test in $7.4 \mathrm{~s}$. For TUG we still found a significant $7 \%$ improvement, independent of training modality. Other studies (Hauer et al. 2001; Kryger and Andersen 2007; Lastayo et al. 2002) in which frail or reconvalescent elderly subjects were trained showed improvements of TUG and leg strength in the order of $50 \%$. We see the failure of the standard risk assessment tools TUG and BBS, to demonstrate large improvements as a consequence of the good physical condition of our subjects at the outset of the study (Lotscher et al. 2007).

Leg strength was assessed in this study by measuring Leg strength developed in a restrained sitting position (MEL). Relative MEL increased significantly by $8.4 \%$ in subjects only after eccentric training. We believe that the small but significant improvement in MEL in EET subjects is biologically relevant. Small gains in maximal performance may cause larger improvements in submaximal performance as a consequence of the non-linear relationship of power versus time or maximal versus repeated activity (Dufour et al. 2006; Wilkie 1985). Maximal performance testing has the advantage of yielding reliable results (Schroeder et al. 2007), while submaximal performance is difficult to assess reliably, but it is more relevant in order to characterize practical benefits for the subjects.

The assessment of body composition showed an unexpected but significant $5 \%$ decrease in body fat content along with a significant $6.9 \%$ decrease in thigh fat content in EET subjects only. Both RET and EET subjects showed a significant increase in relative thigh muscle mass after training. This relative increase was due to an increase in thigh muscle mass (more pronounced with RET), combined with a decrease in thigh fat mass (significant with EET). From these estimates we assume that the observed increase in leg extension strength in EET (reported above) cannot entirely be attributed to a structural change of muscle tissue, but seems to be a consequence of functional (i.e. neural) improvements. It is difficult to assess the potential benefit of a $5 \%$ decrease in body fat content in an elderly population with EET; however, a simultaneous gain of muscle mass with a decrease of body fat counteracts the sarcopenia that usually develops with age.

The muscle fiber type analysis showed two major results: a significant correlation between the type IIX/type II ratio and body composition and a decrease of the type IIX/type II ratio exclusively with EET. The type IIX/type II ratio describes the fraction of the type IIX fibers from the type II fiber pool. It can be assumed that the lower typeIIX/ type II ratio of leaner subjects is related to physical activity and lifestyle. It has previously been described that physical activity in elderly leads to a decrease of type IIX fibers (representing the most anaerobic muscle fiber type) in favor of more aerobic type IIA fibers (Herbison et al. 1982).
Since types I and IIA muscle fibers possess a larger oxidative capacity than type IIX fibers (Herbison et al. 1982) they are better able to couple ATP regeneration with fatty acid and carbohydrate catabolism. In fact, Kriketos et al. (1996) showed a significant positive correlation of relative body fat content with type IIX fibers as well as negative correlations with oxidative enzyme activity (citrate synthase, hexokinase) and insulin sensitivity. This result is in accordance with the limited capacity of type IIX fibers to utilize fatty acids as substrates for ATP regeneration due to their relatively small mitochondrial density (Gueguen et al. 2005). The observed changes of the type IIX/type II ratio with individual changes in body composition parameters indicates the maintenance of plasticity of muscle fibers and body composition as influenced by physical training into old age even at low exercise frequencies.

The higher proportion of type IIX fibers in women may reflect their initial lower fitness possibly due to their less active lifestyle. Due to their greater potential for improvements (strength, coordination) we find women to profit more from training (MEL, eccentric coordination) than men. It has been suggested that muscle fiber composition is implicated in the correlation of body fat content and risk for noninsulin-dependent diabetes mellitus (NIDDM) (Jensen et al. 2007). This view is compatible with our findings, as we find a positive influence of physical training (EET) on fiber composition and potentially reducing the risk of developing agedependent metabolic diseases such as NIDDM.

In conclusion, at the low training frequency of our study EET was similarly successful as RET in improving muscle functional and structural parameters analyzed in this study. EET significantly improved MEL, body composition and eccentric coordination. EET further showed the persistence of muscular plasticity in elderly as evidenced by a decrease of the type IIX/type II muscle fiber ratio. The latter was found to correlate with body composition in all the subjects studied. These findings suggest that eccentric exercise modalities, given their low metabolic costs, merit further evaluation with regard to their potential to improve muscle motor and metabolic functionality in elderly.

Acknowledgments This project was supported by the National Research Programme NRP 53 "Musculoskeletal Health-Chronic Pain" of the Swiss National Science Foundation (Project 405340-104718) and by the University of Bern, Switzerland. A special thank goes to Dunja Minder and Glenn Lurman for editorial proof-reading and to the training instructors from the Federal Office of Sports, Switzerland and the Institute of Sports and Sports Science, University of Bern, Switzerland.

\section{References}

Berg KO, Maki BE, Williams JI, Holliday PJ, Wood-Dauphinee SL (1992) Clinical and laboratory measures of postural balance in an elderly population. Arch Phys Med Rehabil 73:1073-1080 
Bergstrom J (1975) Percutaneous needle biopsy of skeletal muscle in physiological and clinical research. Scand J Clin Lab Invest 35:609-616. doi:10.3109/00365517509095787

Billeter R, Weber H, Lutz H, Howald H, Eppenberger HM, Jenny E (1980) Myosin types in human skeletal muscle fibers. Histochemistry 65:249-259. doi:10.1007/BF00493174

Borg G, Hassmen P, Lagerstrom M (1987) Perceived exertion related to heart rate and blood lactate during arm and leg exercise. Eur $\mathbf{J}$ Appl Physiol Occup Physiol 56:679-685. doi:10.1007/BF00 424810

Doherty TJ (2003) Invited review: aging and sarcopenia. J Appl Physiol 95:1717-1727

Dufour SP, Ponsot E, Zoll J, Doutreleau S, Lonsdorfer-Wolf E, Geny B, Lampert E, Fluck M, Hoppeler H, Billat V, Mettauer B, Richard R, Lonsdorfer J (2006) Exercise training in normobaric hypoxia in endurance runners. I. Improvement in aerobic performance capacity. J Appl Physiol 100:1238-1248. doi:10.1152/japplphysiol.00742.2005

Ericson MO, Nisell R (1986) Tibiofemoral joint forces during ergometer cycling. Am J Sports Med 14:285-290. doi:10.1177/ 036354658601400407

Ericson MO, Nisell R (1987) Patellofemoral joint forces during ergometric cycling. Phys Ther 67:1365-1369

Friden J, Sjostrom M, Ekblom B (1983) Myofibrillar damage following intense eccentric exercise in man. Int J Sports Med 4:170 176. doi:10.1055/s-2008-1026030

Gueguen N, Lefaucheur L, Fillaut M, Herpin P (2005) Muscle fiber contractile type influences the regulation of mitochondrial function. Mol Cell Biochem 276:15-20. doi:10.1007/s11010-005-2464-y

Hauer K, Rost B, Rutschle K, Opitz H, Specht N, Bartsch P, Oster P, Schlierf $G$ (2001) Exercise training for rehabilitation and secondary prevention of falls in geriatric patients with a history of injurious falls. J Am Geriatr Soc 49:10-20. doi:10.1046/j.1532-5415.2001. 49004.x

Herbison GJ, Jaweed MM, Ditunno JF (1982) Muscle fiber types. Arch Phys Med Rehabil 63:227-230

Hersey WC 3rd, Graves JE, Pollock ML, Gingerich R, Shireman RB, Heath GW, Spierto F, McCole SD, Hagberg JM (1994) Endurance exercise training improves body composition and plasma insulin responses in 70- to 79-year-old men and women. Metabolism 43:847-854. doi:10.1016/0026-0495(94)90265-8

Hruda KV, Hicks AL, McCartney N (2003) Training for muscle power in older adults: effects on functional abilities. Can J Appl Physiol 28:178-189

Hungerford DS, Barry M (1979) Biomechanics of the patellofemoral joint. Clin Orthop Relat Res 9-15

Jensen CB, Storgaard H, Madsbad S, Richter EA, Vaag AA (2007) Altered skeletal muscle fiber composition and size precede wholebody insulin resistance in young men with low birth weight. J Clin Endocrinol Metab 92:1530-1534. doi:10.1210/jc.2006-2360

Kaufman KR, An KN, Litchy WJ, Morrey BF, Chao EY (1991) Dynamic joint forces during knee isokinetic exercise. Am J Sports Med 19:305-316. doi:10.1177/036354659101900317

Kriketos AD, Pan DA, Lillioja S, Cooney GJ, Baur LA, Milner MR, Sutton JR, Jenkins AB, Bogardus C, Storlien LH (1996) Interrelationships between muscle morphology, insulin action, and adiposity. Am J Physiol 270:R1332-R1339

Kryger AI, Andersen JL (2007) Resistance training in the oldest old: consequences for muscle strength, fiber types, fiber size, and MHC isoforms. Scand J Med Sci Sports 17:422-430

Langley GB, Sheppeard H (1985) The visual analogue scale: its use in pain measurement. Rheumatol Int 5:145-148. doi:10.1007/ BF00541514

Lastayo PC, Reich TE, Urquhart M, Hoppeler H, Lindstedt SL (1999) Chronic eccentric exercise: improvements in muscle strength can occur with little demand for oxygen. Am J Physiol 276:R611-R615
Lastayo PC, Johns R, Lindstedt SL (2002) Chronic eccentric exercise as a countermeasure for high-fall risk elderly individuals (Abstract)

LaStayo PC, Ewy GA, Pierotti DD, Johns RK, Lindstedt S (2003) The positive effects of negative work: increased muscle strength and decreased fall risk in a frail elderly population. J Gerontol A Biol Sci Med Sci 58:M419-M424

Lexell J (1995) Human aging, muscle mass, and fiber type composition. J Gerontol 50 Spec No:11-16

Lotscher F, Loffel T, Steiner R, Vogt M, Klossner S, Popp A, Lippuner K, Hoppeler H, Dapp C (2007) Biologically relevant sex differences for fitness-related parameters in active octogenarians. Eur J Appl Physiol 99:533-540. doi:10.1007/s00421-006-0368-5

Meyer K, Steiner R, Lastayo P, Lippuner K, Allemann Y, Eberli F, Schmid J, Saner H, Hoppeler H (2003) Eccentric exercise in coronary patients: central hemodynamic and metabolic responses. Med Sci Sports Exerc 35:1076-1082. doi:10.1249/01.MSS. 0000074580.79648.9D

Moritani T, deVries HA (1980) Potential for gross muscle hypertrophy in older men. J Gerontol 35:672-682

Overend TJ, Cunningham DA, Paterson DH, Lefcoe MS (1993) Anthropometric and computed tomographic assessment of the thigh in young and old men. Can J Appl Physiol (Revue Canadienne de Physiologie Appliquee) 18:263-273

Perry MC, Carville SF, Smith IC, Rutherford OM, Newham DJ (2007) Strength, power output and symmetry of leg muscles: effect of age and history of falling. Eur J Appl Physiol 100:553-561. doi:10. 1007/s00421-006-0247-0

Roig M, O’Brien K, Kirk G, Murray R, McKinnon P, Shadgan B, Reid DW (2008) The effects of eccentric versus concentric resistance training on muscle strength and mass in healthy adults: a systematic review with meta-analyses. Br J Sports Med. doi:10.1136/ bjsm.2008.051417

Rooyackers JM, Berkeljon DA, Folgering HT (2003) Eccentric exercise training in patients with chronic obstructive pulmonary disease. Int J Rehabil Res (Internationale Zeitschrift fur Rehabilitationsforschung) 26:47-49

Schroeder ET, Wang Y, Castaneda-Sceppa C, Cloutier G, Vallejo AF, Kawakubo M, Jensky NE, Coomber S, Azen SP, Sattler FR (2007) Reliability of maximal voluntary muscle strength and power testing in older men. J Gerontol A Biol Sci Med Sci 62:543-549

Shigematsu R, Rantanen T, Saari P, Sakari-Rantala R, Kauppinen M, Sipila S, Heikkinen E (2006) Motor speed and lower extremity strength as predictors of fall-related bone fractures in elderly individuals. Aging Clin Exp Res 18:320-324

Shumway-Cook A, Brauer S, Woollacott M (2000) Predicting the probability for falls in community-dwelling older adults using the Timed Up \& Go Test. Phys Ther 80:896-903

Skelton DA, Greig CA, Davies JM, Young A (1994) Strength, power and related functional ability of healthy people aged $65-89$ years. Age Ageing 23:371-377. doi:10.1093/ageing/23.5.371

Steiner R, Meyer K, Lippuner K, Schmid JP, Saner H, Hoppeler H (2004) Eccentric endurance training in subjects with coronary artery disease: a novel exercise paradigm in cardiac rehabilitation? Eur J Appl Physiol 91:572-578. doi:10.1007/s00421-003-1000-6

Vandervoort AA (2002) Aging of the human neuromuscular system. Muscle Nerve 25:17-25. doi:10.1002/mus.1215

Vogt M, Dapp C, Blatter J, Weisskopf J, Suter G, Hoppeler H (2003) Training zur Optimierung der Dosierung exzentrischer Muskelaktivität. Schweizerische Zeitschrift für Sportmedizin und Sporttraumatologie: 188-191

Wieser M, Haber P (2007) The effects of systematic resistance training in the elderly. Int J Sports Med 28:59-65. doi:10.1055/s-2006924057

Wilkie DR (1985) Muscle function: a personal view. J Exp Biol 115:1-13 